\title{
FUNCTION-THEORETIC SOLUTION TO A CLASS OF DUAL INTEGRAL EQUATIONS AND AN APPLICATION TO DIFFRACTION THEORY*
}

\author{
BY
}

ROBERT A. SCHMELTZER (Bell Telephone Laboratories, Holmdel, N. J.) AND MYRNA LEWIN (Courant Institute of Mathematical Sciences, New York University)

Summary. Dual integral equations of the type

$$
\begin{aligned}
\int_{0}^{\infty} u^{\lambda} f(u) J_{\mu}(r u) d u & =g(r), & & 0<r<1, \\
\int_{0}^{\infty} u\left(u^{2}+a^{2}\right)^{-1 / 2} f(u) J_{\nu}(r u) d u & =h(r), & & 1<r<\infty,
\end{aligned}
$$

where $g(r), h(r)$ are prescribed functions and $f(u)$ is to be found, are solved exactly by the application of function-theoretic methods. As an example, a closed-form solution is obtained for the diffraction of an electromagnetic wave by a plane slit.

1. Introduction. A considerable number of boundary-value problems arise in which the boundary conditions are "mixed" in the sense that the unknown function satisfies different types of boundary conditions over distinct portions of the same boundary. An important class of problems of this kind can be reduced to the solution of the dual integral equations of the type

$$
\begin{aligned}
\int_{0}^{\infty} u^{\lambda} f(u) J_{\mu}(r u) d u & =g(r), & & 0<r<1, \\
\int_{0}^{\infty} u\left(u^{2}+a^{2}\right)^{-1 / 2} f(u) J_{\nu}(r u) d u & =h(r), & & 1<r<\infty,
\end{aligned}
$$

where $g(r), h(r)$ are prescribed functions and $f(u)$ is to be found.

These equations were previously solved for a number of special cases. For example, the case in which $a=0, \mu=\nu, \lambda>0, h(r)=0$ was solved by Titchmarsh [1], whose work was later extended by Busbridge [2] and Peters [3]. Dual integral equations of the form (1), (2) arise in the problem of the diffraction of electromagnetic waves by a plane slit. For the case of normal incidence, the problem has been reduced to the solution of (1), (2) with $a=i \epsilon, \epsilon$ real; $\mu=\nu=-\frac{1}{2}, \lambda=1, h(r)=0$. This particular problem was considered for the narrow slit, i.e., $\epsilon \ll 1$ in a paper by Groschwitz and Hönl [4], and later criticized by Tranter [5], Müller and Westpfahl [6]. The exact solution for this problem has not appeared in the literature. Equations (1), (2) also find application in the linearized theory of supersonic flow over a thin wing of infinite span [7], [10].

The method developed here is based on function-theoretic techniques [8] and leads to a closed-form solution of (1), (2) under quite general conditions. It is shown that our solution reduces to the known solution for the case $a=0$. As an example, the solution is applied to the problem of the diffraction of an unsymmetrical electromagnetic wave incident upon a slit of arbitrary width.

2. Reduction of the dual integral equations. We begin by reducing the dual integral

*Received February 28, 1963; revised manuscript received May 13, 1963. This paper presents results obtained at North American Aviation, Los Angeles Division, Los Angeles, Cal. 
equations (1), (2) to a form suitable for the application of function-theoretic techniques. Our method is similar to that employed by Peters. [3]. Our object is to change the orders of the Bessel functions appearing in (1), (2) from $\mu$ and $\nu$ respectively, to $\frac{1}{2}$. This is accomplished in (1) by multiplying each side of (1) by $\left(x^{2}-r^{2}\right)^{p-\mu-1} r^{\mu+1}$ and integrating from 0 to $x$. If it is assumed that the orders of integration of the resulting double integral can be interchanged, we can use a result due to Sonine, namely

$$
\int_{0}^{x}\left(x^{2}-r^{2}\right)^{p-\mu-1} r^{\mu+1} J_{\mu}(u r) d r=2^{p-\mu-1} \Gamma(p-\mu) x^{p} u^{\mu-p} J_{p}(x u), \quad-1<\mu<p,
$$

to obtain

$$
\begin{aligned}
\int_{0}^{\infty} u^{\lambda+\mu-p} f(u) J_{p}(x u) d u & \\
= & \frac{2^{\mu-p+1} x^{-p}}{\Gamma(p-\mu)} \int_{0}^{x}\left(x^{2}-r^{2}\right)^{p-\mu-1} r^{\mu+1} g(r) d r, \quad-1<\mu<p, \quad 0<x<1 .
\end{aligned}
$$

Similarly, multiplying each side of $(2)$ by $\left(r^{2}-x^{2}\right)^{\nu-p-1} r^{1-\nu}$, integrating from $x$ to infinity, and using the result

$$
\begin{aligned}
\int_{x}^{\infty}\left(r^{2}-x^{2}\right)^{\nu-p-1} & r^{1-\nu} J_{\nu}(u r) d r \\
& =2^{\nu-p-1} \Gamma(\nu-p) u^{p-\nu} x^{-p} J_{p}(x u), \quad u>0, \quad p<\nu<2 p+\frac{3}{2},
\end{aligned}
$$

we find

$$
\begin{aligned}
\int_{0}^{\infty} & u^{p-\nu+1}\left(u^{2}+a^{2}\right)^{-1 / 2} f(u) J_{p}(x u) d u \\
& =\frac{2^{p-\nu-1} x^{p}}{\Gamma(\nu-p)} \int_{x}^{\infty}\left(r^{2}-x^{2}\right)^{\nu-p-1} r^{1-\nu} h(r) d r, \quad p<\nu<2 p+\frac{3}{2}, \quad 1<x<\infty .
\end{aligned}
$$

If we choose $p=\frac{1}{2}, \lambda+\mu-1=1-\nu$ and set $\bar{f}(u)=u^{-\nu+1} f(u)$, we have

$$
\begin{aligned}
\int_{0}^{\infty} \bar{f}(u) \sin (x u) d u & =\frac{2^{\mu} \Gamma\left(\frac{1}{2}\right)}{\Gamma\left(\frac{1}{2}-\mu\right)} \int_{0}^{x}\left(x^{2}-r^{2}\right)^{-\mu-1 / 2} r^{1+\mu} g(r) d r, \quad 0<x<1, \\
\int_{0}^{\infty}\left(u^{2}+a^{2}\right)^{-1 / 2} \bar{f}(u) \sin (x u) d u & \\
= & \frac{2^{1-\nu} \Gamma\left(\frac{1}{2}\right) x}{\Gamma\left(\nu-\frac{1}{2}\right)} \int_{x}^{\infty}\left(r^{2}-x^{2}\right)^{\nu-3 / 2} r^{1-\nu} h(r) d r, \quad 1<x<\infty,
\end{aligned}
$$

subject to the restrictions

$$
-1<\mu<+\frac{1}{2} \text { and } \frac{1}{2}<\nu<\frac{5}{2} \text {. }
$$

The range of $\mu$ and $\nu$ specified in (9) may be altered by multiplying (1), (2) by appropriate powers of $r$ and then differentiating and/or integrating under the integral sign, applying the formulae

$$
\left(\frac{1}{r} \frac{d}{d r}\right)^{m} r^{p+m} J_{p+m}(r u)=u^{m} r^{p} J_{p}(r u),
$$




$$
\left(\frac{1}{r} \frac{d}{d r}\right)^{m} r^{-p-m} J_{p+m}(r u)=(-1)^{m} u^{m} r^{-p} J_{p}(r u),
$$

and subsequently changing the order of the Bessel functions to $\frac{1}{2}$.

Consider for example, the case when $\mu=\nu=\beta<\frac{1}{2}$, for which the restriction on $\nu$ imposed by (9) is violated. Using (10) with $m=1$, we can write (2) in the form

$$
\frac{d}{d r} \int_{0}^{\infty}\left(u^{2}+a^{2}\right)^{-1 / 2} f(u) r^{\nu+1} J_{\nu+1}(r u) d u=r^{\nu+1} h(r),
$$

which upon integrating becomes

$$
\int_{0}^{\infty}\left(u^{2}+a^{2}\right)^{-1 / 2} f(u) J_{\nu+1}(r u) d u=-r^{-\nu-1} \int_{r}^{\infty} t^{\nu+1} h(t) d t .
$$

We can now apply Sonine's integral as above, so that the order of the Bessel function in (13) is changed from $\nu+1$ to $\frac{1}{2}$. If we do this we obtain an alternative equation for (6)

$$
\begin{aligned}
\int_{0}^{\infty} u^{-\nu-1}\left(u^{2}+a^{2}\right)^{-1 / 2} f(u) & \sin (x u) d u \\
= & \frac{-2^{-\nu} \Gamma\left(\frac{1}{2}\right) x}{\Gamma\left(\nu+\frac{1}{2}\right)} \int_{x}^{\infty}\left(r^{2}-x^{2}\right)^{\nu-1 / 2} r^{-2 \nu-1} \int_{r}^{\infty} t^{\nu+1} h(t) d t d r
\end{aligned}
$$

and is valid if $-\frac{1}{2}<\nu<3 / 2,1<x<\infty$.

Therefore, when $-1<\mu<\frac{1}{2}, \frac{1}{2}<\nu<5 / 2$, the dual integral equations (1), (2) are transformed to (7), (8). For $-1<\mu<\frac{1}{2},-\frac{1}{2}<\nu<3 / 2$, equations (1), (2) are transformed to (7) and (14). Similar procedures can be employed to transform (1), (2) for other values of $\mu$ and $\nu$. The value of $\lambda$ is chosen so that the dual integral equations can be written in the form

$$
\begin{array}{ll}
\int_{0}^{\infty} \varphi(u) \sin (x u) d u=q(x), & 0<x<1, \\
\int_{0}^{\infty} \frac{\varphi(u) \sin (x u)}{\left(u^{2}+a^{2}\right)^{1 / 2}} d u=r(x), & 1<x<\infty .
\end{array}
$$

There is no loss of generality if it is assumed that $r(x)=0$. This is not necessary but will simplify the labor of solving (15), (16). To show this, we let

$$
\varphi(u)=\varphi_{0}(u)+\varphi_{1}(u)
$$

where $\varphi_{1}(u)$ satisfies

$$
\int_{0}^{\infty} \frac{\varphi_{1}(u) \sin (x u)}{\left(u^{2}+a^{2}\right)^{1 / 2}} d u= \begin{cases}0, & 0 \leq x<1, \\ r(x), & 1<x<\infty .\end{cases}
$$

Equations (15) and (16) then reduce to the form

$$
\begin{aligned}
& \int_{0}^{\infty} \varphi_{0}(u) \sin (x u) d u \\
& =q(x)-\frac{2}{\pi} \int_{0}^{\infty} \sin (x u)\left(u^{2}+a^{2}\right)^{1 / 2} \int_{1}^{\infty} r(t) \sin (u t) d t d u, \quad 0 \leq x<1, \\
& \quad \int_{0}^{\infty} \frac{\varphi_{0}(u) \sin (x u)}{\left(u^{2}+a^{2}\right)^{1 / 2}} d u=0, \quad 1<x<\infty .
\end{aligned}
$$


3. Solution of dual integral equations. We proceed to the solution of the dual integral equations (15), (16) with $r(x)=0$. Equation (15) is satisfied by

$$
\varphi(u)=\frac{2}{\pi} \int_{0}^{\infty} \psi(t) \sin (u t) d t
$$

provided that

$$
\psi(x)=q(x), \quad 0 \leq x<1
$$

where $\psi(x)$ is assumed Lebesgue integrable $L(0, \infty)$ and Hölder continuous in the intervals $0<x<1,1<x<\infty$. If (21) is substituted into (16) and the order of integration of the resulting double integral changed, we obtain the integral equation

$$
\frac{2}{\pi} \int_{0}^{\infty} \psi(t) \int_{0}^{\infty} \frac{\sin (t u) \sin (x u)}{\left(u^{2}+a^{2}\right)^{1 / 2}} d u d t=0, \quad 1<x<\infty .
$$

The inner integral is uniformly convergent for all values of $x$ and its value is given by Watson [9]. Hence (16) can be written in the form

$\frac{1}{\pi} \int_{0}^{\infty} \psi(t)\left\{K_{0}(a|x-t|)-K_{0}(a|x+t|)\right\} d t=0$,

$$
1<|x|<\infty, \quad|\arg a| \leq \pi / 2
$$

where $K_{0}$ is the modified Bessel function of the third kind of order zero. Using (22), we obtain the integral equation for $\psi(t), 1<|t|<\infty$

$\frac{1}{\pi} \int_{1}^{\infty} \psi(t)\left\{K_{0}(a|x-t|)-K_{0}(a|x+t|)\right\} d t=-G(x), \quad 1<|x|<\infty$,

where

$$
G(x)=\frac{1}{\pi} \int_{0}^{1} q(t)\left\{K_{0}(a|x-t|)-K_{0}(a|x+t|)\right\} d t, \quad 0<|x|<\infty .
$$

Consider the function $F(Z)$ defined by

$$
F(Z)=\frac{d}{d Z} \int_{1}^{\infty} \psi(t)\left\{K_{0}[a(t-Z)]-K_{0}[a(t+Z)]\right\} d t
$$

for the sector $-2 \pi<\arg Z<-\pi$. The kernel of (27) is of the form

$$
\begin{aligned}
K_{0}[a(t-Z)]-K_{0}[a(t+Z)]=-\log ( & t-Z) I_{0}[a(t-Z)] \\
& +\log (t+Z) I_{0}[a(t+Z)]+Q(Z, t),
\end{aligned}
$$

where $I_{0}$ is the modified Bessel function of the first kind of order zero, and $Q$ is an entire function of $Z . F(Z)$ can be analytically continued to an analytic function on a Riemann surface having branch points at $Z= \pm 1$. To show this, define a branch cut on the line $1<R(Z)<\infty$ such that $K_{0}[a(t-Z)]$ is analytic in the sector $-2 \pi<\arg Z<0$ or

$$
-\pi<\arg (t-Z)<+\pi \text {. }
$$

Define a second branch cut on $-\infty<R(Z)<-1$ such that $K_{0}[a(t+Z)]$ is analytic in the sector $-\pi<\arg Z<\pi$, or

$$
-\pi<\arg (t+Z)<\pi .
$$


If we write $F(Z)$ in the form

$$
\begin{aligned}
F(Z)=\frac{d}{d Z} \int_{1}^{x} \psi(t)\left\{K_{0}[a(t-Z)]\right. & \left.-K_{0}[a(t+Z)]\right\} d t \\
& +\frac{d}{d Z} \int_{x}^{\infty} \psi(t)\left\{K_{0}[a(t-Z)]-K_{0}[a(t+Z)]\right\} d t
\end{aligned}
$$

and define

$$
\begin{array}{ll}
F^{+}(x)=\lim _{I(Z) \rightarrow 0^{+}} F(Z) & R(Z)=x, \\
F^{-}(x)=\lim _{I(Z) \rightarrow 0^{-}} F(Z) & R(Z)=x,
\end{array}
$$

then it is readily verified that for $x$ real,

$$
\begin{aligned}
\frac{1}{2}\left[F^{+}(x)+F^{-}(x)\right]=\frac{d}{d x} \int_{1}^{\infty} \psi(t)\left\{K_{0}(a|t-x|)-K_{0}(a|t+x|)\right\} d t, & 0<|x|<\infty .
\end{aligned}
$$

The right hand side of (34) is known for $1<|x|<\infty$ from (26) and may be analytically continued off the real axis by replacing $x$ by $Z=x+i y$. The modulus signs in the argument of the $K_{0}$ functions in (26) can be removed in the interval $1<x<\infty$ and $G(x)=-G(-x)$. Hence $G(x)$ is analytic and can be continued from the real axis $1<|x|<\infty$ into the complex $Z$-plane. Equating the right hand side of (34) to the analytic function of (26), we obtain

$$
F^{+}(x)+F^{-}(x)=-2 \pi G^{\prime}(x), \quad 1<|x|<\infty
$$

providing a relation for analytically continuing $F(Z)$ to an analytic function on all sheets of a Riemann surface having branch points at $Z= \pm 1$. Since $F(Z)$, as defined by the integral (27), is continuous for all $|Z|<1$, we also have

$$
F^{+}(x)-F^{-}(x)=0, \quad 0<|x|<1
$$

Equations (35) and (36) define a Hilbert problem for the sectionally holomorphic function $F(Z)$ which can be determined uniquely and explicitly provided the behavior of $F(Z)$ is prescribed at infinity. The behavior of $F(Z)$ at infinity can be determined from the definition integral (27).

Lemma 1. $\quad F(Z)$ as defined by (27) satisfies

$$
F(Z)=O\left(|Z|^{-1 / 2} \cosh a Z\right), \quad|I(Z)|>0
$$

as $Z \rightarrow \infty$.

Proof. Consider the function $F_{1}(Z)$, analytic in the sector $-2 \pi<\arg Z<0$, and defined by the integral

$$
F_{1}(Z)=\frac{d}{d Z} \int_{1}^{\infty} \psi(t) K_{0}[a(t-Z)] d t, \quad|I(Z)|>0 .
$$

First observe that in order to assure convergence of (21)

$$
\lim _{t \rightarrow \infty} \psi(t)=0 .
$$

Define an arbitrary large constant $R$ such that for $t \geq R, 1 \ll R \ll x,|\psi(t)| \leq 1$, then

$$
\left|F_{1}(Z)\right| \leq\left|\frac{d}{d Z} \int_{1}^{R} \psi(t) K_{0}[a(t-Z)] d t\right|+\left|K_{0}[a(R-Z)]\right|, \quad|I(Z)|>0 .
$$


Since $K_{0}(Z)=O\left(|Z|^{-1 / 2} \exp -Z\right)$ at infinity, we find

$$
F_{1}(Z)=O\left(|Z|^{-1 / 2} \exp a Z\right), \quad|I(Z)|>0, \quad \text { as } \quad Z \rightarrow \infty .
$$

Observing that $F(Z)$ as defined by (27) is given by

$$
F(Z)=F_{1}(Z)+F_{1}(-Z)
$$

we have proven Lemma 1.

Lemma 2. The most general solution for a sectionally holomorphic function $F(Z)$ as defined by (27), satisfying the boundary conditions (35), (36) and Lemma 1 is of the form

$$
F(Z)=[A(Z) \exp (-a Z)-A(-Z) \exp (+a Z)] / Z,
$$

where $A(Z)$ is itself sectionally holomorphic, of order

$$
A(Z)=O\left(|Z|^{1 / 2}\right)
$$

at infinity and satisfying the boundary conditions

$$
\begin{array}{lr}
A^{+}(x)-A^{-}(x)=0, & 0 \leq|x|<1, \\
A^{+}(x)+A^{-}(x)=\left\{\begin{array}{lr}
0, & -\infty<x<-1, \\
-2 \pi x G^{\prime}(x) \exp (a x), & 1<x<\infty .
\end{array}\right.
\end{array}
$$

Proof. Let $F(Z)$ be written in the form

$$
F(Z)=[A(Z) \exp (-a Z)+B(Z) \exp (+a Z)] / Z,
$$

where $A(Z), B(Z)$ are taken as arbitrary functions of $Z$. Equation (37) implies that

$$
B(Z)=-A(-Z)
$$

and that $A(Z), B(Z)$ are analytic for $|I(Z)|>0$. It follows from Lemma 1 that

$$
A(Z)=O\left(|Z|^{1 / 2}\right) \text { as }|Z| \rightarrow \infty
$$

To complete the proof, we show that $A(Z)$ satisfies the boundary conditions (39), (40). In view of (35), we have for $x>1$,

$$
\begin{aligned}
F^{+}(x)+F^{-}(x) & =\left[\left\{A^{+}(x)+A^{-}(x)\right\} \exp (-a x)-\left\{A^{+}(-x)+A^{-}(-x)\right\} \exp (a x)\right] / x \\
& =-2 \pi G^{\prime}(x) .
\end{aligned}
$$

Upon examination of (26) it is observed that

$$
G^{\prime}(x)=0\left(|x|^{-1 / 2} \exp (-a|x|)\right), \text { as }|x| \rightarrow \infty .
$$

This implies that $A^{+}(-x)+A^{-}(-x)=0$, thereby verifying (40). Since $F(Z)$ is continuous for $|Z|<1$

$$
A^{+}(x)-A^{-}(x)=0, \quad 0<|x|<1,
$$

which establishes the validity of Lemma 2 .

We have therefore reduced the problem to that of finding a sectionally holomorphic 
function $A(Z)$ of order $0\left(|Z|^{1 / 2}\right)$ at infinity and satisfying the boundary conditions (39), (40). The solution for $A(Z)$ proceeds as follows. Let

$$
A(Z)=A_{0}(Z) A_{1}(Z)
$$

where $A_{0}(Z)$ satisfies the homogeneous problem, obtained from (39), (40) by setting $G^{\prime}(x)=0$, i.e., we choose $A_{0}(Z)$ to be a sectionally holomorphic function, of finite degree at infinity, and satisfying

$$
\begin{array}{ll}
A_{0}^{+}(x)+A_{0}^{-}(x)=0, & 1<|x|<\infty, \\
A_{0}^{+}(x)-A_{0}^{-}(x)=0, & 0<|x|<1 .
\end{array}
$$

A particular solution of (44), (45) is $\left(1-Z^{2}\right)^{-1 / 2}$. The general solution is of the form $A_{0}(Z)=P_{0}(Z)\left(1-Z^{2}\right)^{-1 / 2}, \quad-\pi<\arg (1-Z)<\pi, \quad-\pi<\arg (1+Z)<\pi$,

where $P_{0}(Z)$ is an arbitrary function of finite degree at infinity. It will now be shown that $P_{0}(Z)$ is in fact, an arbitrary polynomial. To show this, let $A_{0}(Z)$ be any solution, and $X(Z)$ be a particular solution vanishing nowhere in the finite part of the plane including the real axis. Then

$$
A_{0}^{+}(x)+A_{0}^{-}(x)=0, \quad X^{+}(x)+X^{-}(x)=0, \quad 1<|x|<\infty,
$$

and hence, since $X^{+}(x) \neq 0, X^{-}(x) \neq 0$,

$$
A_{0}^{+}(x) / X^{+}(x)=A_{0}^{-}(x) / X^{-}(x)
$$

Consequently, the function $A_{0}(Z) / X(Z)$ is holomorphic in the whole plane. Since it has a finite degree at infinity it is a polynomial and the statement is proved.

Consequently $A_{1}(Z)$ satisfies

$$
A_{1}^{+}(x)-A_{1}^{-}(x)= \begin{cases}-2 \pi x G^{\prime}(x) \exp (a x) / A_{0}^{+}(x), & 1<x<\infty, \\ 0, & 0<|x|<1,-\infty<x<-1,\end{cases}
$$

where

$$
A_{0}^{+}(x)=+i P_{0}(x)\left(x^{2}-1\right)^{-1 / 2}, \quad 1<x<\infty .
$$

The most general solution of (47) is of the form

$$
A_{1}(Z)=\int_{1}^{\infty} \frac{i x G^{\prime}(x) \exp (a x)}{A_{0}^{+}(x)(x-Z)} d x+P_{1}(Z),
$$

where $P_{1}(Z)$ is an arbitrary polynomial. Substituting (46), (48) and (49) into (43) yields the most general solution for $A(Z)$,

$$
A(Z)=\frac{P_{0}(Z)}{\left(1-Z^{2}\right)^{1 / 2}} \int_{1}^{\infty} \frac{x\left(x^{2}-1\right)^{1 / 2} G^{\prime}(x) \exp (a x)}{P_{0}(x)(x-Z)} d x+\frac{P_{0}(Z) P_{1}(Z)}{\left(1-Z^{2}\right)^{1 / 2}}
$$

Lemma 3. The polynomial $P_{0}(Z)$ must be of second degree, i.e., of the form

$$
P_{0}(Z)=C\left(Z^{2}+\alpha Z+\beta\right),
$$

where $\alpha, \beta$ and $C$ are arbitrary constants, $C \neq 0$. The polynomial $P_{1}(Z)$ is identically zero. 
Proof. Let $n$ and $m$ denote the respective orders of $P_{0}(Z)$ and $P_{1}(Z)$ at infinity, i.e., $P_{0}(Z)=0\left(Z^{n}\right), P_{1}(Z)=0\left(Z^{m}\right)$ as $Z \rightarrow \infty$. Equation (50) implies that

$$
\begin{aligned}
|A(Z)| \leq \mid C Z^{n-1} \int_{1}^{R} & \frac{x\left(x^{2}-1\right)^{1 / 2} G^{\prime}(x) \exp (a x)}{P_{0}(x)(x-Z)} d x \mid \\
& +\left|C Z^{n-1} \int_{R}^{\infty} \frac{x\left(x^{2}-1\right)^{1 / 2} G^{\prime}(x) \exp (a x)}{P_{0}(x)(x-Z)} d x\right|+\left|\frac{P_{0}(Z) P_{1}(Z)}{\left(1-Z^{2}\right)^{1 / 2}}\right|
\end{aligned}
$$

where $R$ is an arbitrary large constant such that $1 \ll R<\infty$. Then for $x>R$

$$
\left|\frac{C x\left(x^{2}-1\right)^{1 / 2} G^{\prime}(x) \exp (a x)}{P_{0}(x)}\right| \leq\left|C_{1} x^{3 / 2-n}\right| .
$$

This follows directly from the asymptotic behavior of $G^{\prime}(x)$ as given by (42). Hence

$$
|A(Z)| \leq C_{0}|Z|^{n-2}+\left|C_{1} Z^{n-1} \int_{R}^{\infty} \frac{x^{3 / 2-n}}{x-Z} d x\right|+\left|C_{2} Z^{n+m-1}\right| \text { as } Z \rightarrow \infty .
$$

In order to secure convergence, $n \geq 2$. Thus

$$
\begin{aligned}
& |A(Z)| \leq C_{0}|Z|^{n-2}+\left|C_{1} \int_{R}^{\infty} \frac{Z}{x^{1 / 2}(x-Z)} d x-\sum_{m=1}^{n-2} \int_{R}^{\infty} \frac{Z^{m}}{x^{m+1 / 2}} d x\right|+\left|C_{2} Z^{n+m-1}\right|, \\
& n \geq 2, \text { as } Z \rightarrow \infty \\
& \begin{array}{r}
|A(Z)|<C_{0}|Z|^{n-2}+C_{1}|Z|^{1 / 2}\left|\pi-2 \tan ^{-1}(R / Z)^{1 / 2}-\sum_{m=1}^{n-2} \frac{(Z / R)^{m-1 / 2}}{m-\frac{1}{2}}\right| \\
+\left|C_{2} Z^{n+m-1}\right| .
\end{array}
\end{aligned}
$$

Hence

$$
A(Z)=0\left(Z^{n-2}\right)+0\left(Z^{1 / 2}\right)+0\left(Z^{n+m-1}\right), \quad n \geq 2 \quad \text { as } \quad Z \rightarrow \infty .
$$

Since $n \leq 2$ from Lemma 2, $P_{0}(Z)$ must be of second degree at infinity, and $P_{1}(Z) \equiv 0$.

We have therefore shown $F(Z)$ is given by

$$
\begin{aligned}
F(Z)= & \frac{\left(1-Z^{2}\right)^{-1 / 2}}{Z} \\
& \cdot \int_{1}^{\infty} \frac{x\left(x^{2}-1\right)^{1 / 2} G^{\prime}(x)}{P_{0}(x)}\left[\frac{P_{0}(Z) \exp a(x-Z)}{x-Z}-\frac{P_{0}(-Z) \exp a(x+Z)}{x+Z}\right] d x \\
& -\pi<\arg (1+Z)<\pi, \quad-\pi<\arg (1-Z)<\pi,
\end{aligned}
$$

where $P_{0}(Z)$ is an arbitrary polynomial of the form stated in Lemma 3. Sufficient conditions for determining $F(Z)$ uniquely is considered in Theorem 1.

Theorem 1. Let $\psi(x)$ be Hölder continuous in the interval $0 \leq x<\infty$ and $q(x)$ Hölder continuous in the closed interval $0 \leq x \leq 1$. Then $P_{0}(Z)$ is given by $P_{0}(Z)=$ $C\left(Z^{2}-1\right)$, where $C$ is an arbitrary nonvanishing constant, $\varphi(u)$ is uniquely determined

$$
\begin{aligned}
\varphi(u) & =\frac{2}{\pi} \frac{\left(u^{2}+a^{2}\right)^{1 / 2}}{u} \int_{0}^{1} G^{\prime}(x) \cos (u x) d x \\
& -\frac{2}{\pi^{2}} \frac{\left(u^{2}+a^{2}\right)^{1 / 2}}{u} \int_{0}^{1} \frac{\left(1-x^{2}\right)^{1 / 2}}{x} \cos (u x) \int_{1}^{\infty} \frac{t G^{\prime}(t)}{\left(t^{2}-1\right)^{1 / 2}}\left\{\frac{e^{a(t-x)}}{(t-x)}-\frac{e^{a(t+x)}}{(t+x)}\right\} d t d x,
\end{aligned}
$$


and

$$
G^{\prime}(t)=\frac{1}{\pi} \frac{d}{d t} \int_{0}^{1} q(s)\left\{K_{0}(a|s-t|)-K_{0}(a|s+t|)\right\} d s .
$$

Proof. Consider the behavior of the function $F(Z)$ as $Z$ approaches the branch point $Z=1$. Let $R$ denote a real positive constant. If $R$ is chosen sufficiently close to unity, then for $R>|Z|, F(Z)$ as defined by (27) behaves as

$$
F(Z)=-\frac{d}{d Z} \int_{1}^{R} \psi(t) \log (t-Z) d t+O(1) \quad \text { as } \quad Z \rightarrow 1 .
$$

The integral in (53) is evaluated as

$$
\begin{aligned}
-\frac{d}{d Z} \int_{1}^{R} \psi(t) \log (t-Z) d t & =\int_{1}^{R} \frac{\psi(t)-\psi(1)}{t-Z} d t+\psi(1) \int_{1}^{R} \frac{d t}{t-Z} \\
& =-\psi(1) \log (1-Z)+O(1)
\end{aligned}
$$

in view of the assumed Hölder continuity of $\psi(t)$. Hence

$$
F(Z)=-\psi(1) \log (1-Z)+O(1) \quad \text { as } Z \rightarrow 1 .
$$

Now $\psi(x)$ is related to $q(x)$ by the formula

$$
q(x)=\frac{2}{\pi} \int_{0}^{\infty} \sin (u x) \int_{0}^{\infty} \psi(t) \sin (u t) d t d u
$$

obtained by substituting (21) into (15). Since $\psi(t)$ is Hölder continuous and belongs to $L(0, \infty), \psi(x)=q(x)$ in the closed interval $0 \leq x \leq 1$. Therefore

$$
F(Z)=-q(1) \log (1-Z)+O(1) \text { as } Z \rightarrow 1 .
$$

It will now be shown that (54) is sufficient for determining $P_{0}(Z)$ uniquely. $F(Z)$ as given by (52) may be shown to behave as

$$
F(Z)=\frac{P_{0}(Z)}{\left(1-Z^{2}\right)^{1 / 2}} \int_{1}^{\infty} \frac{\left(x^{2}-1\right)^{1 / 2} G^{\prime}(x)}{P_{0}(x)(x-Z)} d x+\frac{P_{0}(-Z)}{\left(1-Z^{2}\right)^{1 / 2}} O(1)+O(1) \text { as } Z \rightarrow 1 \text {. }
$$

Using the fact that $G^{\prime}(x)=q(1) \log (x-1)+O(1)$ as $x \rightarrow 1$, we find

$$
F(Z)=O(q(1) \log (1-Z))+O\left(\frac{P_{0}(Z)}{\left(1-Z^{2}\right)^{1 / 2}}\right)+O\left(\frac{P_{0}(-Z)}{\left(1-Z^{2}\right)^{1 / 2}}\right)+O(1) \text { as } Z \rightarrow 1
$$

In order to satisfy (54) it is necessary that

$$
\frac{P_{0}(Z)}{\left(1-Z^{2}\right)^{1 / 2}} \text { and } \frac{P_{0}(-Z)}{\left(1-Z^{2}\right)^{1 / 2}}
$$

be bounded at $Z=1$. The only polynomial of second degree satisfying this condition is $P_{0}(Z)=C\left(Z^{2}-1\right)$, where $C$ is an arbitrary constant, $C \neq 0$. Therefore, $F(Z)$ is uniquely determined

$$
\begin{aligned}
F(Z)=-\frac{\left(1-Z^{2}\right)^{1 / 2}}{Z} \int_{1}^{\infty} & \frac{x G^{\prime}(x)}{\left(x^{2}-1\right)^{1 / 2}}\left\{\frac{\exp a(x-Z)}{x-Z}-\frac{\exp a(x+Z)}{x+Z}\right\} d x, \\
& -\pi<\arg (1-Z)<\pi, \quad-\pi<\arg (1+Z)<\pi .
\end{aligned}
$$


It is now a simple matter to find $\varphi(u)$. We have shown that (16) can be written in the form given by (24), viz.,

$$
\int_{0}^{\infty} \frac{\varphi(u) \sin x u}{\left(u^{2}+a^{2}\right)^{1 / 2}} d u=\frac{1}{\pi} \int_{0}^{\infty} \psi(t)\left\{K_{0}(a|t-x|)-K_{0}(a|t+x|)\right\} d t .
$$

Using (34) and (26),

$$
\frac{d}{d x} \int_{0}^{\infty} \frac{\varphi(u) \sin x u}{\left(u^{2}+a^{2}\right)^{1 / 2}} d u=G^{\prime}(x)+\frac{1}{2 \pi}\left\{F^{+}(x)+F^{-}(x)\right\}, \quad 0<|x|<\infty .
$$

Now $F^{+}(x)+F^{-}(x)$ can be found from (56); thus

$$
\begin{aligned}
& \frac{d}{d x} \int_{0}^{\infty} \frac{\varphi(u) \sin x u}{\left(u^{2}+a^{2}\right)^{1 / 2}} d u \\
& \quad= \begin{cases}G^{\prime}(x)-\frac{1}{\pi} \frac{\left(1-x^{2}\right)^{1 / 2}}{x} \int_{1}^{\infty} \frac{t G^{\prime}(t)}{\left(t^{2}-1\right)^{1 / 2}}\left[\frac{e^{a(t-x)}}{t-x}-\frac{e^{a(t+x)}}{t+x}\right] d t, & 0<x<1 \\
0, & 1<x<\infty .\end{cases}
\end{aligned}
$$

The Fourier cosine transform of (59) yields the result sought

$$
\begin{aligned}
\frac{\pi}{2} \frac{u \varphi(u)}{\left(u^{2}+a^{2}\right)^{1 / 2}} & =\int_{0}^{1} G^{\prime}(x) \cos (u x) d x \\
& -\frac{1}{\pi} \int_{0}^{1} \frac{\left(1-x^{2}\right)^{1 / 2}}{x} \cos (u x) \int_{1}^{\infty} \frac{t G^{\prime}(t)}{\left(t^{2}-1\right)^{1 / 2}}\left\{\frac{e^{a(t-x)}}{t-x}-\frac{e^{a(t+x)}}{t+x}\right\} d t d x
\end{aligned}
$$

which proves Theorem 1.

Remark. Equation (60) is uniformly valid for $0 \leq a<\infty,|\arg a| \leq \pi / 2$. In fact, for the special case $a=0$, the solution for $\varphi(60)$ reduces to

$$
\varphi(u)=\frac{2 u}{\pi} \int_{0}^{1} t J_{1}(u t) \int_{0}^{1} q(r t) r\left(1-r^{2}\right)^{-1 / 2} d r d t
$$

(see Appendix). This is Titchmarsh's formula for the solution of the equations

$$
\begin{aligned}
\int_{0}^{\infty} y^{\alpha} f(y) J_{\nu}(x y) d y=g(x), & 0<x<1, \\
\int_{0}^{\infty} f(y) J_{\nu}(x y) d y=0, & 1<x<\infty
\end{aligned}
$$

for the case $\alpha=1, \nu=\frac{1}{2}$, where $f(y)=y^{-1 / 2} \varphi(y) ; g(x)=(2 / \pi x)^{1 / 2} q(x)$.

4. An application to a diffraction problem. As an example of these results, consider the problem of the diffraction of an $E$-polarized electromagnetic wave by an infinite conducting plane with a slit of arbitrary width. Let the slit lie in the $y=0$ plane of a right handed Cartesian coordinate system and be bounded by the lines $x= \pm b$. The remaining portion of the plane, viz., $y=0,|x|>b$, consists of a perfectly conducting plate of zero thickness. We consider the case in which the fields are independent of the $z$ coordinate and the electric field vector is parallel to the $z$-axis, i.e., $H_{z}=E_{x}=E_{\nu}=0$. Maxwell's equations reduce to

$$
\frac{\partial E_{z}^{\prime}}{\partial y}=i \mu \omega H_{x}: \quad \frac{\partial E_{z}}{\partial x}=-i \mu \omega H_{y}: \quad \frac{\partial H_{y}}{\partial x}-\frac{\partial H_{x}}{\partial y}=-i \epsilon \omega E_{z}^{\prime}
$$


where $\epsilon$ is the dielectric constant and $\mu$ is the permeability. The electric field satisfies the wave equation

$$
\frac{\partial^{2} \varphi}{\partial x^{2}}+\frac{\partial^{2} \varphi}{\partial y^{2}}+k^{2} \varphi=0 ; \quad k^{2}=\epsilon \mu \omega^{2}
$$

Denote the total field in the presence of the slit by

$$
\varphi_{i}(x, y)=\varphi_{i}(x, y)+\varphi(x, y)
$$

where $\varphi(x, y)$ is the sum of the reflected and diffracted waves. In the absence of the conducting plate, the incident wave is given by

$$
\varphi_{i}(x, y)=\exp (-i k x \cos \theta-i k y \sin \theta), \quad 0<\theta<\pi
$$

where $\theta$ is the angle between the negative $x$-axis and the direction of propagation of the incident wave.

The function $\varphi(x, y)$ is chosen to satisfy the radiation-condition at infinity and the boundary condition $\varphi_{t}=0$ on the perfectly conducting plate but not in the slit. Currents are induced in the plate in the $z$ direction, i.e.,

$$
I_{z}=\left(H_{x}\right)_{0+}-\left(H_{x}\right)_{0-}=(i \mu \omega)^{-1}\left[\left(\frac{\partial E_{z}}{\partial y}\right)_{0+}-\left(\frac{\partial E_{z}}{\partial y}\right)_{0-}\right] .
$$

Because no currents can flow in the slit, we have

$$
\left(\frac{\partial \varphi_{t}}{\partial y}\right)_{0+}=\left(\frac{\partial \varphi_{l}}{\partial y}\right)_{0-} \quad|x|<b .
$$

Using the condition that the electric field must be continuous across $y=0,0<|x|<\infty$, the total field $\varphi_{t}$ can be represented in the form

$$
\begin{aligned}
\varphi_{\iota}(x, y) & =\int_{-\infty}^{\infty} U(p) \exp [+\gamma y-i p x] d p, & & y \leq 0 \\
& =\int_{-\infty}^{\infty} U(p) \exp [-\gamma y-i p x] d p+\varphi_{i}(x, y)-\varphi_{i}(x,-y), & & y \geq 0
\end{aligned}
$$

where $\gamma=\left(p^{2}-k^{2}\right)^{1 / 2}$ and $\varphi_{i}(x, y)$ is given by (66).

Since $\varphi_{t}=0$ on $y=0, b<|x|<\infty$, we find that the function $U(p)$ must satisfy

$$
\int_{-\infty}^{\infty} U(p) \exp (-i p x) d p=0, \quad b<|x|<\infty
$$

and by $(67)$

$$
\int_{-\infty}^{\infty} \gamma U(p) \exp (-i p x) d p=-i k \sin \theta \exp (-\imath k x \cos \theta), \quad 0<|x|<b .
$$

Equations (69), (70) represent a set of dual integral equations for the unknown function $U(p)$. These equations are also applicable to the problem of an $H$-polarized electromagnetic wave incident on a conducting strip of arbitrary width, as well as to the analogous acoustic problems.

These equations can be reduced to two independent sets of dual integral equations having sine and cosine kernels and of a form suitable for solution. Let

$$
U(p)=U_{\bullet}(p)+U_{0}(p),
$$


where

$$
U_{.}(p)=\frac{1}{2}[U(p)+U(-p)] \text {, }
$$

$$
U_{0}(p)=\frac{1}{2}[U(p)-U(-p)]
$$

Then (69), (70) reduce to

$$
\begin{array}{rlrl}
\int_{0}^{\infty} U_{0}(p)\left(p^{2}-k^{2}\right)^{1 / 2} \sin p x d p & =\frac{-i k}{2} \sin \theta \sin (k x \cos \theta), & & 0 \leq|x|<b, \\
\int_{0}^{\infty} U_{0}(p) \sin p x d p & =0, & & b<|x|<\infty, \\
\int_{0}^{\infty} U_{0}(p)\left(p^{2}-k^{2}\right)^{1 / 2} \cos p x d p=\frac{-i k}{2} \sin \theta \cos (k x \cos \theta), & & 0 \leq|x|<b, \\
\int_{0}^{\infty} U_{0}(p) \cos p x d p=0, & & b \leq|x|<\infty .
\end{array}
$$

With

$$
\begin{gathered}
u=p b, \quad x^{\prime}=x / b, \quad \epsilon=k b, \\
\varphi_{\bullet}(u)=k U_{e}(u / b)\left(u^{2}-\epsilon^{2}\right)^{1 / 2}, \quad \varphi_{0}(u)=k U_{0}(u / b)\left(u^{2}-\epsilon^{2}\right)^{1 / 2},
\end{gathered}
$$

Equations (74), (75), (76), (77) become

$$
\begin{array}{ll}
\int_{0}^{\infty} \varphi_{0}(u) \sin x^{\prime} u d u=\frac{-i \epsilon^{2}}{2} \sin \theta \sin \left(\epsilon x^{\prime} \cos \theta\right), & 0<\left|x^{\prime}\right|<1, \\
\int_{0}^{\infty} \frac{\varphi_{0}(u) \sin x^{\prime} u}{\left(u^{2}-\epsilon^{2}\right)^{1 / 2} d u=0,} & 1<\left|x^{\prime}\right|<\infty, \\
\int_{0}^{\infty} \varphi_{e}(u) \cos x^{\prime} u d u=\frac{-i \epsilon^{2}}{2} \sin \theta \cos \left(\epsilon x^{\prime} \cos \theta\right), & 0<\left|x^{\prime}\right|<1, \\
\int_{0}^{\infty} \frac{\varphi_{e}(u) \cos x^{\prime} u}{\left(u^{2}-\epsilon^{2}\right)^{1 / 2}} d u=0, & 1<\left|x^{\prime}\right|<\infty .
\end{array}
$$

This problem was considered for the case of a narrow slit, i.e., $\epsilon \ll 1$ and $\theta=\pi / 2$ by Groschwitz and Hönl [4] whose work was later criticized by Tranter [5], Müller and Westpfahl [6]. The complete solution of these equations can be determined in closed form for slits of arbitrary width by applying the results of the previous section with $a=\epsilon e^{-i \pi / 2}$. The solution of dual integral equations (78), (79) is given by

$\frac{u \pi \varphi_{0}(u)}{2\left(u^{2}-\epsilon^{2}\right)^{1 / 2}}=\frac{1}{2} \pi u k U_{0}(u / b)=\int_{0}^{1} G_{0}^{\prime}(x) \cos u x d x$

$-\frac{1}{\pi} \int_{0}^{1} \frac{\left(1-x^{2}\right)^{1 / 2}}{x} \cos u x \int_{1}^{\infty} \frac{t G_{0}^{\prime}(t)}{\left(t^{2}-1\right)^{1 / 2}}\left[\frac{\exp [-i \epsilon(t-x)]}{t-x}-\frac{\exp [-i \epsilon(t+x)]}{t+x}\right] d t d x$,

where

$$
G_{0}^{\prime}(t)=\frac{1}{4} \epsilon^{2} \sin \theta \frac{d}{d t} \int_{0}^{1} \sin (\epsilon x \cos \theta)\left\{H_{0}^{(1)}[\epsilon|x-t|]-H_{0}^{(1)}[\epsilon|\dot{x}+t|]\right\} d x
$$

and $H_{0}^{(1)}(Z)$ is the Bessel function of the third kind,

$$
H_{0}^{(1)}(Z)=\frac{2}{\pi i} K_{0}\left(Z e^{-i \pi / 2}\right) .
$$


The solution of the dual integral equations (80), (81) proceeds as follows. If (80) is integrated from 0 to $r$ and (81) is integrated from 1 to $r$, we obtain

$$
\begin{aligned}
\int_{0}^{\infty} \varphi_{e}(u) \frac{\sin r u}{u} d u & =\frac{-i \epsilon}{2} \tan \theta \sin (\epsilon r \cos \theta), & & 0 \leq r<1, \\
\int_{0}^{\infty} \varphi_{e}(u) \frac{\sin r u}{u\left(u^{2}-\epsilon^{2}\right)^{1 / 2}} d u & =C^{\prime}=0, & & 1<r<\infty .
\end{aligned}
$$

The integration constant $C^{\prime}$ in (85) must be zero. For if we assume $\varphi_{0}(u) u^{-1}\left(u^{2}-\epsilon^{2}\right)^{-1 / 2}$ is Lebesgue integrable, $L(0, \infty)$ then the integral in (85) must tend to zero as $r \rightarrow \infty$. Using (60) we find

$\frac{\pi}{2} \frac{\varphi_{e}(u)}{\left(u^{2}-\epsilon^{2}\right)^{1 / 2}}=\frac{\pi}{2} k U_{\bullet}(u / b)=\int_{0}^{1} G_{\bullet}^{\prime}(x) \cos u x d x$

$-\frac{1}{\pi} \int_{0}^{1} \frac{\left(1-x^{2}\right)^{1 / 2}}{x} \cos u x \int_{1}^{\infty} \frac{t G_{e}^{\prime}(t)}{\left(t^{2}-1\right)^{1 / 2}}\left\{\frac{\exp \left[-i_{\epsilon}(t-x)\right]}{t-x}-\frac{\exp \left[-i_{\epsilon}(t+x)\right]}{t+x}\right\} d t d x$,

where

$$
G_{0}^{\prime}(t)=\frac{1}{4} \epsilon \tan \theta \frac{d}{d t} \int_{0}^{1} \sin (\epsilon x \cos \theta)\left\{H_{0}^{(1)}[\epsilon|x-t|]-H_{0}^{(1)}[\epsilon|x+t|)\right\} d x .
$$

5. Appendix. We shall here show that our solution reduces to that given by Titchmarsh [1] for the special case $a=0$. The dual integral equations (15), (16) become

$$
\begin{aligned}
\int_{0}^{\infty} \varphi(u) \sin x u d u & =q(x), & & 0 \leq x<1, \\
\int_{0}^{\infty} u^{-1} \varphi(u) \sin x u d u & =0, & & 1<x<\infty .
\end{aligned}
$$

The solution to the dual integral equations (15), (16) given by (60) is uniformly valid for $0 \leq a<\infty,|\arg a| \leq \pi / 2$. In fact, for the case $a=0$, (60) reduces to $\varphi(u)=-\frac{2}{\pi} \int_{0}^{1} G^{\prime}(x) \cos u x d x$

$$
-\frac{4}{\pi^{2}} \int_{0}^{1}\left(1-x^{2}\right)^{1 / 2} \cos u x \int_{1}^{\infty} t\left(t^{2}-1\right)^{-1 / 2} \frac{G^{\prime}(t)}{t^{2}-x^{2}} d t d x,
$$

where $G^{\prime}(t)$ is obtained from (26) with $a=0$,

$$
G^{\prime}(t)=\frac{2}{\pi} \int_{0}^{1} \frac{r q(r)}{r^{2}-t^{2}} d r
$$

Substituting (A4) into (A3) and interchanging orders of integration we obtain

$$
\begin{aligned}
\varphi(u)=\frac{4}{\pi^{2}} \int_{0}^{1} \cos u x \int_{0}^{1} \frac{r q(r)}{r^{2}-x^{2}} d r d x \\
\quad-\frac{8}{\pi^{3}} \int_{0}^{1}\left(1-x^{2}\right)^{1 / 2} \cos u x \int_{0}^{1} r q(r) \int_{1}^{\infty} \frac{t\left(t^{2}-1\right)^{1 / 2}}{\left(t^{2}-x^{2}\right)\left(r^{2}-t^{2}\right)} d r d x .
\end{aligned}
$$


The last integral is easily evaluated and (A5) reduces to

$$
\varphi(u)=\frac{4}{\pi^{2}} \int_{0}^{1}\left(1-x^{2}\right)^{1 / 2} \cos u x \int_{0}^{1} \frac{r q(r)}{\left(r^{2}-x^{2}\right)\left(1-r^{2}\right)^{1 / 2}} d r
$$

which can be manipulated into the form

$$
\varphi(u)=\frac{4}{\pi^{2}} \int_{0}^{1} x\left(1-x^{2}\right)^{-1 / 2} \frac{\sin x u}{u} \int_{0}^{1} \frac{\left(1-r^{2}\right)^{1 / 2}}{x^{2}-r^{2}} q^{\prime}(r) d r d x .
$$

Next we substitute

$$
\frac{\sin x u}{u}=\int_{0}^{x} t J_{0}(u t)\left(x^{2}-t^{2}\right)^{-1 / 2} d t
$$

into (A7) and interchange orders of integration:

$$
\varphi(u)=\frac{4}{\pi^{2}} \int_{0}^{1}\left(1-r^{2}\right)^{1 / 2} q^{\prime}(r) \int_{0}^{1} t J_{0}(u t) \int_{t}^{1} \frac{x\left(1-x^{2}\right)^{-1 / 2}\left(x^{2}-t^{2}\right)^{-1 / 2}}{x^{2}-r^{2}} d x d t d r .
$$

Hence

$$
\varphi(u)=\frac{2}{\pi} \int_{0}^{1} q^{\prime}(r) \int_{r}^{1} \frac{t J_{0}(u t)}{\left(t^{2}-r^{2}\right)^{1 / 2}} d t d r .
$$

If the order of integration in (A10) be changed, this can be written as

$$
\varphi(u)=\frac{\iota}{\pi} \int_{0}^{1} J_{0}(u t) \frac{d}{d t} \int_{0}^{t} q^{\prime}(r)\left(t^{2}-r^{2}\right)^{1 / 2} d r d t
$$

If we integrate the second integral by parts noting that $q(0)=0$ we obtain

$$
\varphi(u)=\frac{2}{\pi} \int_{0}^{1} J_{0}(u t) \frac{d}{d t} \int_{0}^{t} \frac{r q(r)}{\left(t^{2}-r^{2}\right)^{1 / 2}} d r d t .
$$

Integrating the first integral by parts then gives

$$
\varphi(u)=\frac{2 u}{\pi} \int_{0}^{1} t J_{1}(u t) \int_{0}^{1} r\left(1-r^{2}\right)^{-1 / 2} q(r t) d r d t+\frac{2}{\pi} J_{0}(u) \int_{0}^{1} t q(t)\left(1-t^{2}\right)^{-1 / 2} d t .
$$

which agrees identically with Titchmarsh's result provided

$$
\int_{0}^{1} t\left(1-t^{2}\right)^{-1 / 2} q(t) d t=0 .
$$

To show that (A14) holds true, multiply (A1) by $x\left(1-x^{2}\right)^{-1 / 2}$ and integrate from 0 to 1 . If we change orders of integration in the resulting double integral and use the formula

$$
\int_{0}^{1} \frac{x \sin x u}{\left(1-x^{2}\right)^{1 / 2}} d x=\frac{1}{2} \pi J_{1}(u)
$$

we obtain

$$
\int_{0}^{1} x q(x)\left(1-x^{2}\right)^{-1 / 2} d x=\frac{\pi}{2} \int_{0}^{\infty} \varphi(u) J_{1}(u) d u .
$$

The right hand side of (A16) can be determined from (A2) by assuming one sided continuity at $x=1$ of the integral in (A2), $1 \leq x<\infty$. To show this, multiply (A2) 
by $\left(x^{2}-r^{2}\right)^{-1 / 2}$ and integrate from 0 to $\infty$. If we interchange the orders of integration and use

$$
\int_{r}^{\infty}\left(x^{2}-r^{2}\right)^{-1 / 2} \sin x u d x=\frac{\pi}{2} J_{0}(r u)
$$

we find

$$
\int_{0}^{\infty} \frac{\varphi(u)}{u} J_{0}(r u) d u=0, \quad 1<r<\infty .
$$

Differentiation then yields

$$
\int_{0}^{\infty} \varphi(u) J_{1}(r u) d u=0, \quad 1<r<\infty .
$$

Thus if the integral appearing in (A2) is continuous $1 \leq x<\infty$, then

$$
\int_{0}^{1} r q(r)\left(r^{2}-t^{2}\right)^{-1 / 2} d r=\frac{\pi}{2} \int_{0}^{\infty} \varphi(u) J_{1}(u) d u=0 .
$$

\section{REFERENCES}

1. E. C. Titchmarsh, Introduction to the theory of Fourier integrals; Oxford, 1937, p. 337

2. I. W. Busbridge, Dual integral equations, Proc. London Math. Soc. 44 (1938) 115-29

3. A. S. Peters, Certain dual integral equations and Sonine's integrals, New York University Report, IMM-285 (1961)

4. E. Groschwitz and H. Honl, Diffraction of electromagnetic waves by a slit, Zeit. f. Physik 131 (1952), 305-19

5. C. J. Tranter, $A$ further note on dual integral equations and an application to the diffraction of electromagnetic waves, Quart. Journ. Mech. Applied Math. 7 (1954) 317-25

6. R. Müller and K. Westpfahl, $A$ rigorous treatment of the diffraction of electromagnetic waves by a slit, Zeits. f. Physik 134 (1953) 245-63

7. J. C. Gunn, Linearized supersonic aerofoil theory, Phil. Trans. A240 (1947) 327

8. A. E. Heins and R. C. MacCamy, A function-theoretic solution of certain integral equations (I), Quart. J. Math., (2) 9 (1958) 132-43

9. G. N. Watson, Bessel functions, Cambridge, 1934

10. M. Lewin and R. A. Schmeltzer, A new method for treating supersonic flow past nearly plane wings, to appear in J. Fluid Mechanics 